\title{
W. F. Dagajew: erste experimentelle Untersuchungen der Magenresektion
}

Wladimir Fjodorowitsch Dagajew (1872-1958) ist als ein großer Organisator im Bereich des Gesundheitswesens in die Geschichte eingegangen. Dagajew wurde 1872 im Dorf Spasskoje im Nowossilsk-Kreis des Tula-Gouvernements in einer Priesterfamilie geboren. Nach dem Abschluss des Priesterseminars in Tula begann er 1896 sein Studium an der Medizinischen Fakultät der Universität Tomsk, das er 1902 abschloss. Bereits während der Studienzeit begeisterte er sich für die Chirurgie und nach dem Universitätsabschluss widmete er sich der chirurgischen Praxis. Er vertiefte seine chirurgischen Kenntnisse in der Klinik prominenter sibirischer Chirurgen wie E. G. Salischtschew und N. A. Rogowitsch. Eine Zeit lang arbeitete er gemeinsam mit N. A. Bogoraz.

1910 bis 1911 Dagajew war Praktikant am Institut für experimentelle Medizin in Sankt Petersburg, wo er seine Promotionsarbeit unter dem Titel „Zur Lehre vom Verdauungschemismus nach der Teilresektion und der kompletten Entfernung des Magens“ vorbereitete. 1911 wurde er an der Medizinischen Militärakademie promoviert; nach der erfolgreichen Promotion wurde ihm der akademische Grad „Dr. med.“ zuerkannt. 1912 wurde Dagajew von der Rotkreuz-Gesellschaft nach Bulgarien delegiert, um den Verwundeten während des Krieges gegen die Türkei Hilfe zu leisten. 1913 wurde er zum Chefarzt und Leiter der chirurgischen Abteilung im Altaischen Krankenhaus der Eisenbahn bestellt (Nowonikolajewsk - heute Nowosibirsk); später bekleidete er die gleichen Ämter im Krankenhaus in Mariupol.

Von 1914 bis zur Pensionierung im Jahre 1957 arbeitete Dagajew ununterbrochen in Tula. Seit 1914 leitete er die Spitäler des Gemeindeverbandes des Tula-Gouvernements mit dem Hauptspital am Gebietskrankenhaus Tula. 1924 wurde er zum Chefarzt und Leiter der chirurgischen Abteilung im neu gegründeten N. A. Semaschko Stadtkrankenhaus in Tula bestellt. Zu dieser Einrichtung gehörte auch das chirurgische Krankenhaus als „seine“ Abteilung. Unter der Leitung von Dagajew wurde das Stadtkrankenhaus wesentlich umgebaut und erweitert.

D. A. Balalykin, Russische Errungenschaften in der Physiologie der Verdauung, https://doi.org/10.1007/978-3-662-62044-1_3 
Von 1931 bis 1938 fungierte die von Dagajew geleitete chirurgische Abteilung des Stadtkrankenhauses Tula als zweite Chirurgieklinik des Zentralen Instituts für Weiterbildung der Ärzte, die er als wissenschaftlicher Betreuer leitete.

Für seine Verdienste im Bereich des Gesundheitswesens wurde ihm der Ehrentitel „Verdienter Arzt der RSFSR“1 verliehen. 1952 wurde zu Ehren seines 80. Geburtstages und seines 50-jährigen ärztlichen und gesellschaftlichen Engagements dem Chirurgieblock des N. A. Semaschko Stadtkrankenhauses in Tula mit Erlass des Ministers der RSFSR für das Gesundheitswesen der Name „W. F. Dagajew“ gegeben. Dagajew starb am 7. Februar 1958 in Tula, wo auch seine Gebeine ruhen.

Aus dem Curriculum vitae, das der Promotionsarbeit beigelegt ist, ergibt sich, dass Dagajew die Doktorprüfungen in den Jahren 1906/07 ablegte und seit September 1910 als Praktikant am Kaiserlichen Institut für experimentelle Medizin in Sankt Petersburg tätig war. Auf der Titelseite der Dissertation ist angegeben, dass zu den „Zensoren“ (,Opponenten“ in der heutigen Terminologie) Akademiemitglied I. P. Pawlow, Professor M. D. Iljin und Privatdozent W. N. Tomaschewski gehörten.

Uns gelang es, einige Dokumente zum Promotionsverfahren Dagajews zu finden. ${ }^{2}$ In einem mit 2.11.1911 datierten Dokument steht es: „Die Kommission, zu der Professoren und Akademiemitglied Pawlow I. P., Iljin M. D. und Privatdozent Tomaschewski gehören, behandelte die Promotionsarbeit von Wladimir Fjodorowitsch Dagajew unter dem Titel „Zur Lehre vom Verdauungschemismus nach der Teilresektion und der kompletten Entfernung des Magens"3 und befand sie zufriedenstellend und für die Veröffentlichung zulässig ${ }^{4}$.

Von Pawlow wurde handschriftlich folgendes Dokument abgefasst und von den Kommissionsmitgliedern unterzeichnet: „Gegen die Festlegung der Promotion der Dissertation des Arztes W. F. Dagajew unter dem Titel „Zur Lehre vom Verdauungschemismus nach der Teilresektion und der kompletten Entfernung des Magens " für Donnerstag, 8. Dezember habe ich keine Einwände." 5 Nach dem Einholen der Zustimmung der Kommission auf der Sitzung der Konferenz der Kaiserlichen Medizinischen Militärakademie am 3. Dezember 1911 Nr. 6 wurde beschlossen: „24. Die Promotionen der Ärzte Geschelin A. I. und Dagajew W. F. werden auf den 8. Dezember ab 3 1/2 Uhr nachmittags festgelegt.“6

\footnotetext{
${ }^{1}$ RSFSR - Russische Sozialistische Föderative Sowjetrepublik. Die größte Teilrepublik der UdSSR.

${ }^{2}$ Später wurden diese Archivakten zum ersten Mal in der „Anthologie der Geschichte der russischen Chirurgie" publiziert. Siehe: Anthologie der Geschichte der russischen Chirurgie, Bd. 2. ${ }^{3}$ Chemismus - ein veralteter Ausdruck. Im modernen Sinne handelt es sich um die Auswertung der Verdauung im Magen und Zwölffingerdarm mit Hilfe von Methoden der analytische Chemie.

${ }^{4}$ Russisches Staatliches Militär-historisches Archiv, Besitz 316, Register 42, Fach 954, S. 7.

${ }^{5}$ Russisches Staatliches Militär-historisches Archiv, Besitz 316, Register 42, Fach 954, S. 7.

${ }^{6}$ Protokolle der Sitzungen der Konferenz der Kaiserlichen Medizinischen Militärakademie für 1911-1912, Sankt Petersburg, 1912, S. 65.
} 
Die Disputation über die Promotionsarbeit von Dagajew fand an der Medizinischen Militärakademie an diesem Tag statt.

Im Protokoll der Konferenzsitzung der Medizinischen Militärakademie vom 10. Dezember 1911 steht geschrieben:

„3. Der wissenschaftliche Sekretär berichtete, dass am 8. Dezember d. J. die Ärzte Geschelin A. I. und Dagajew W. F. promovierten. Die Teilnehmer der Disputation der Kommission bewerteten die Promotionen als zufriedenstellend und erkannten den erwähnten Ärzten den Grad ,Doktor der Medizin`zu.

Beschlossen: die ordentlichen Diplome sind auszuhändigen“.7

Die Dissertation von Dagajew genießt in zwei Hinsichten zweifellos eine Vorrangstellung.

Erstens, wie es der Verfasser der Dissertation selbst betonte, war es die erste Studie, die der Untersuchung der Verdauungsfunktionen bei fehlendem Pylorus und fehlendem Magen, d. h. unter pathologischen Bedingungen diente, ,nachdem Pawlow die wichtigsten Grundsätze des Funktionierens der Verdauungsdrüsen unter den normalen Bedingungen festgestellt hatte. ${ }^{\text {} 8}$

Zweitens forderte die umfassende Untersuchung der digestiven Funktionen neben dem Studium der Funktionen der Verdauungsdrüsen auch, den Einfluss dieser Funktionen auf einzelne Nahrungsarten zu erforschen. Aus diesem Grunde hatte Pawlow festgestellt: „Die komplette Antwort auf die zwei angeführten Fragengruppen, wozu und auf welche Weise die Funktion der Drüsen variiert, werden wir nur dann erhalten, wenn an das Verfahren der Untersuchung der Trennungsvorgänge eine ausführliche Untersuchung des Inhalts des Verdauungstrakts während der gesamten Verdauungsperiode an jedem seinem Punkt geknüpft wird; dann werden wir genau wissen, wo sich welcher Bestandteil der Nahrung befindet und welchen Veränderungen er zum jeweiligen Zeitpunkt ausgesetzt ist. “9

Der Untersuchung eben dieser Aspekte der Verdauung ist die Dissertation von Dagajew gewidmet. Für die Lösung der Aufgaben, vor denen er stand, benutzte er sowohl physiologisch-operative als auch chemische Methoden.

Der methodische Teil der Dissertation ist recht ausführlich, deshalb werden wir nur auf ihre wichtigsten Aspekte hinweisen. Insgesamt führte Dagajew 200 Versuche an neun Hunden durch. Davon waren zwei Kontrolltiere. Bei dem einen (,Woltschok“) wurde eine Fistel im Fundus-Bereich, bei dem anderen (,Belka“) eine enterale Fistel $125 \mathrm{~cm}$ oberhalb der ileozökalen Klappe angelegt. Bei den drei Hunden „Druschok“, „Bobik“ und „Kaschtan“ wurde eine Pylorusresektion mit anschließender Anastomose des Magens und des Zwölffingerdamms nach Kocher vorgenommen. Einem Hund aus dieser

${ }^{7}$ Protokolle der Sitzungen der Konferenz der Kaiserlichen Medizinischen Militärakademie für 1911-1912, Sankt Petersburg, 1912, S. 75.

${ }^{8}$ Anthologie der Geschichte der russischen Chirurgie, Bd. II, S. 57.

${ }^{9}$ Anthologie der Geschichte der russischen Chirurgie, Bd. I, S. 236. 
Gruppe („Druschok“) wurde eine Magenfistel angelegt, zwei weiteren Hunden („Bobik“ und „Kaschtan“) eine Darmfistel, die $125 \mathrm{~cm}$ oberhalb der ileozökalen Klappe lag.

$\mathrm{Zu}$ der zweiten Versuchsgruppe gehörten auch die drei Hunde „Scholty“, „Schawka“ und „Werny“. Bei ihnen wurde eine Magenresektion nach dem Billroth-II-Verfahren vorgenommen. „Scholty“ wurde die Magenfistel, „Schawka“ und „Werny“ die Dünndarmfistel angelegt. Dem Hund „Maltschik“ wurde der gesamte Magen entfernt; für die Verbindung mit dem Zwölffingerdarm wurde der Murphy-Knopf verwendet. Diesem Hund wurde ebenfalls eine Darmfistel angelegt.

Für die Bestimmung der Funktionen einzelner Organe des gastrointestinalen Systems, des Verlaufs des gesamten Digestionsprozesses sowie des Verdauungsgrades einzelner Nahrungsstoffe wurden entsprechende Methoden verwendet, die in der Dissertation ausführlich beschrieben sind. Für die Bestimmung des Verdauungsprozesses wurde zum einen seine „Extensität“ untersucht, d. h. die Menge des sezernierten Chymus im Lauf einer bestimmten Zeit und in bestimmten Stunden der Verdauung, seine Azidität bzw. Alkalität; zum zweiten seine „Intensität“, d. h. der Wirkungsgrad der Verdauung sowie des Abbaus der wichtigsten Nahrungskomponenten. Dabei erkannte Dagajew, anknüpfend an die Hinweise Pawlows, dass ,,bei jeder Nahrung sich sowohl die Menge als auch die Qualität des Saftes mit jeder Stunde in typischer Weise ändert. Beim Fleisch ist die maximale Sekretion einmal in der ersten, ein anderes Mal in der zweiten Stunde nachweisbar; dabei unterscheiden sich diese Zeitintervalle recht wenig hinsichtlich der Menge; beim Brot erscheint das Maximum schlagartig in der ersten Stunde, bei der Milch in der zweiten und sogar in der dritten Stunde. “10

Das gesamte Funktionieren des Verdauungssystems hängt in vielerlei Hinsicht von der Geschwindigkeit ab, mit der der Magen die Nahrung in den Darm transportiert.

Für die Untersuchung der motorischen Leistung und der Entleerung der Nahrungsmasse aus dem Magen unternahm Dagajew spezielle Versuche sowohl am „normalen“ Hund „Woltschok“ als auch an den pylorektomierten Hunden. Auf der Grundlage der Daten, die zur damaligen Zeit in der Forschung vorlagen, formulierte er drei Faktoren, welche die Geschwindigkeit der Evakuation des Chymus aus dem Magen bestimmen:

a) die Menge der in den Magen eingeführten Nahrung;

b) die Zeit, die seit dem Beginn der Nahrungseinführung vergangen ist;

c) der vom Zwölffingerdarm ausgehende Reflex, der im Zusammenhang mit der reflektorischen Einwirkung des Magensaftes auf das Duodenum entsteht.

Ausgehend von der strikten Zweckmäßigkeit, die im Funktionieren des Verdauungstraktes zu sehen ist, findet Dagajew heraus, dass die Leistung, die der Magen bei der Nahrungsentleerung entwickelt, sich sowohl an die Menge des zu evakuierenden Inhalts als auch an die Evakuationszeit anpasst. Für die Bestimmung der Nahrungsmenge,

\footnotetext{
${ }^{10}$ Anthologie der Geschichte der russischen Chirurgie, Bd. I, S. 119-120.
} 
die zum jeweiligen Zeitpunkt im Organ verbleibt, und der Evakuation entwickelte er die folgende Gesetzmäßigkeit, die er in einer mathematischen Formel zum Ausdruck brachte:

$$
x=\mathrm{k} \sqrt{\frac{m}{\mathrm{t}+p}}
$$

Dabei gilt:

„X“ - die Menge der im Magen zum jeweiligen Zeitpunkt verbliebenen Nahrung;

„t“ - die nach der Nahrungseinführung vergangene Zeit;

„p“ - die Menge des in den Darm gelangten Magensaftes.

Die Menge der im Magen zum jeweiligen Zeitpunkt gebliebenen Nahrung (, $\mathrm{x}^{“}$ ) wird sich im umgekehrten Verhältnis zur Zeit (,t“) verringern; zugleich wird sie im umgekehrten Verhältnis zur Menge des in den Zwölffingerdarm gelangenden Magensaftes stehen.

$\mathrm{m}-100 \%$ der in den Magen eingeführten Nahrung;

$\mathrm{k}$ - eine gewisse konstante Größe.

Der Untersuchung eben dieser Aspekte der Verdauung ist die Dissertation von Dagajew gewidmet. Für die Lösung der Aufgaben, vor denen er stand, benutzte er sowohl physiologisch-operative als auch chemische Methoden.

Für die Untersuchung der motorischen Funktionen des Magens bei dem normalen und bei dem operierten Hund benutzte Dagajew eine 5-prozentige Lösung reinen Zuckers. Bekanntlich wird der Zucker im Magen chemisch nicht verarbeitet und deshalb erfüllt dieses Organ hier rein motorische Funktionen. Die Versuche, die der Wissenschaftler an dem nicht operierten Hund durchführte, zeigten, dass die motorische Magenfunktion hauptsächlich nach der von ihm entwickelten Formel verläuft. Die Versuche an dem Hund „Druschok“, bei dem eine Pylorektomie vorgenommen wurde, zeigten, dass bei diesem Hund die Evakuation in der gleichen Weise wie im Normalfall, nur mit verlangsamtem Tempo verläuft. Die Konstante $k$ betrug bei „Woltschok“ 10,35 und bei „Druschok“ 13,6. Bei „Woltschok“ ging die Entleerung des Magens mit der Zuckerlösung nach 60 min fast zu Ende und im Magen verblieben nur $2 \%$ der Lösung; bei „Druschok“ blieben nach 60 min $14 \%$ Zucker und nach 75 min sogar noch $7 \%$ Zucker übrig. Der Gesamtcharakter des Prozesses der Nahrungsentleerung aus dem Magen ist bei beiden Hunden der gleiche, aber bei dem operierten Tier verläuft dieser Prozess wesentlich langsamer.

$\mathrm{Zu}$ jener Zeit, als Dagajew seine Forschungen durchführte, war bekannt, dass der Transport der Nahrung aus dem Magen in den Darm durch die Kontraktionen der Pylorusmuskulatur infolge des vom Zwölffingerdarm ausgehenden Reflexes geregelt wird (I. P. Pawlow, A. S. Sserdjukow, S. I. Lintwarjow, N. Kanzelson, Hirsch, Mering u. a.). Daher wurde angenommen, dass die chirurgische Entfernung des Pylorus große Veränderungen bei allen Teilfunktionen der Magenverdauung auslösen würde. 
Im Labor Pawlows gewann sein Kollege Doktor I. Edelman die Erkenntnis, dass die periodischen Kontraktionen des Magenbodens recht kräftig sind und unabhängig vom Pylorusbereich erfolgen.

Diese Frage untersuchte Dagajew sehr eingehend an den Hunden, denen der Pylorus operativ entfernt worden war. Beim Vergleich des Evakuationsverlaufs des Mageninhalts bei dem nicht operierten Hund mit den pylorektomierten Hunden gelangt er zu dem Schluss, dass , die Nerven, die die Gesetzmäßigkeiten der Magenentleerung steuern, eine selbstständige Grundlage im Fundusbereich des Magens haben“. Dabei erfolgt die Entleerung aus dem Magen ohne Pylorus „,nach der gleichen mathematisch formulierten Gesetzmäßigkeit wie auch die Entleerung aus dem normalen Organ. Der verbliebene Fundus bleibt dabei vom hemmenden Reflex aus dem Zwölffingerdarm unberührt.“

Dagajew untersuchte ferner die Rolle des Reflexes vom Zwölffingerdarm auf den Magenfundus. Nach der Magenresektion nach dem Billroth-I-Verfahren und der Gastrostomie wurde an dem Hund eine zusätzliche Operation durchgeführt. Es wurde ihm eine Fistel im oralen Teil des Zwölffingerdarms angelegt. Mit ihrer Hilfe war es möglich, die Geschwindigkeit des Übertritts der Zuckerlösung aus dem Magen in den Darm unter normalen und - sofern ins Duodenum saure Stoffe eingeführt wurden experimentellen Bedingungen zu verfolgen. Dabei wurde festgestellt, dass im zweiten Fall die Evakuation aus dem pyloruslosen Magen verlangsamt ist. „Sobald“, so Dagajew, „,der Reflex des Zwölffingerdarms auf den Magenpförtner des normalen Magens ausgelöst wird, wird er auch auf den Fundus des Magens ohne Pylorus wirksam. Dieser Reflex bewirkt eine Verzögerung des Beginns und eine Verlangsamung der gesamten Geschwindigkeit der Magenentleerung. "11

Nach der Untersuchung der Chymusevakuation aus dem Magen im normalen Zustand und nach Pylorektomie gelangt er zur folgenden Schlussfolgerung: „Der Magen, der keinen Pylorusteil besitzt und durch Anastomose direkt mit dem Zwölffingerdarm verbunden ist, wird von den Zuckerlösungen wesentlich langsamer als der normale Magen befreit. Die Evakuation des pyloruslosen Magens erfolgt nach der gleichen mathematisch formulierten Gesetzmäßigkeit wie auch die Entleerung des normalen Magens. Der verbliebene Magenfundus bleibt dabei von dem hemmenden Reflex des Zwölffingerdarms nicht vollkommen frei. Bei den Zuckerlösungen mit höherer Konzentration fließen in den Magen transpylorische Säfte (so bezeichnete Dagajew den Inhalt des Zwölffingerdarms der Verf.), die die alkalische Reaktion seines Inhalts bedingen." ${ }^{2}$

Die Versuche zur Verdauung der Kohlenhydrate im Magen nach der Pylorektomie wurden mit Amylodextrin durchführt. Diese Untersuchungen wurden am Hund im Anschluss an die Magenresektion nach Billroth-I durchgeführt. Bekanntlich wird Amylodextrin im Magen des Hundes im Normalfall nicht abgebaut, weil in seinem Speichel Ptyalin fehlt. Es wird ausschließlich im Darm abgebaut.

\footnotetext{
${ }^{11}$ Anthologie der Geschichte der russischen Chirurgie, Bd. 2, S. 86.

${ }^{12}$ Anthologie der Geschichte der russischen Chirurgie, Bd. 2, S. 88.
} 
An „Woltschok“ und „Druschok“ wurden jeweils elf Versuche unternommen. In zwei Versuchsserien wurde die 5-prozentige Amylodextrinlösung in einer Menge von 200 und $600 \mathrm{~cm}^{3}$ in den Magen der Hunde eingeführt. Im nicht operierten Magen ist der Zucker gar nicht enthalten, im teilresezierten Magen ist in allen Versuchen der freie Zucker vorhanden. Die Reaktion des Mageninhalts ist aufgrund des Übertritts der ,transpylorischen Säfte“ in den Magen immer alkalisch. Der Chemismus der Verdauung selbst ändert sich auch: Im Magen des operierten Hundes erfolgt jene Verdauungsfunktion, die in einer normalen Situation nur für den mittleren Darmbereich typisch ist.

Bei der Diskussion der Ergebnisse, die während der Fütterung des nach BillrothI operierten Hundes mit Fett zustande kamen, geht Dagajew von den zur damaligen Zeit vorhandenen Angaben aus (I. P. Pawlow, P. P. Chischin, I. O. Lobassow, T. M. Wirschubski, W. N. Boldyrew), nach denen die Sekretion des Magensaftes bei fetthaltiger Nahrung unterdrückt wird, seine Azidität sinkt und der Übertritt von Gallenflüssigkeit und Pankreassaft in den Magen zu beobachten ist. Ähnliche Daten erhielt Dagajew auch in seinen Versuchen an dem nicht operierten Hund. Das Gewicht des Mageninhalts blieb in allen Versuchen bei recht niedrigen Werten; niedrig war auch die Azidität. Das Chymusgewicht war bei dem nicht operierten Hund höher als bei dem aus der Kontrollgruppe und stieg mit der zunehmenden Dauer der Verdauung. Dies beweist den Übertritt des Inhalts des Zwölffingerdarms in den Magen, was günstige Voraussetzungen für Fettverdauung schafft. Der Magen, der keinen Pylorusteil hat, befreit sich von den Fettstoffen langsamer als der normale. Bei motorischer Verlangsamung der Verdauung wird ihre Intensität wesentlich erhöht, darauf weisen gesteigerte Prozesse des Fettabbaus und der Fettaufspaltung hin.

Bei der reinen Eiweißverdauung, die bei der Verabreichung von Fleisch an den Hund zu beobachten ist, erhielt Dagajew folgende Ergebnisse: Wenn dem normalen Hund Hackfleisch verabreicht wurde, wurde dieses innerhalb von drei Stunden im Wesentlichen verdaut. Binnen drei Stunden hat der Magen eine beträchtliche Menge Fleisch in den Darm weitertransportiert. Unter den analogen Verhältnissen wurde bei dem operierten Hund Fleisch einer geringeren chemischen Verarbeitung ausgesetzt, und in den Darm gelangte eine geringere Fleischmenge. Bei allen Versuchen verblieb im operierten Magen fast doppelt so viel Stickstoff wie im normalen. Aus allen Versuchen mit Fleisch zieht Dagajew die Schlussfolgerung, dass der Magen des operierten Hundes seine Arbeit etwa um die Hälfte langsamer als der normale verrichtet.

Bei den Versuchen mit Fleisch blieb die Reaktion des Mageninhalts sauer, was beweist, dass es in diesem Falle entweder gar keinen Reflux des Inhalts des Zwölffingerdarms in den Magen gab oder dieser unwesentlich war und die alkalische Reaktion des Mageninhalts nicht ändern konnte.

Die Versuche mit Zucker, Amylodextrin und Schweinefett wurden mit der gleichen Nahrung durchgeführt. Dagajew untersuchte die Verdauungsfunktion des Magens ohne Pylorus auch bei der Verabreichung einer Nahrung, die viele lebensnotwendige chemische Stoffe enthielt. Als solche Nahrung gilt für den Hund vor allem Brot. Aus den Schriften der Mitarbeiter von Pawlow (P. P. Chischin, I. O. Lobassow) war bekannt, 
dass als Reaktion auf die Gabe von Brot eine geringere Magensaftsekretion beobachtet wird als bei Fleisch. In den Versuchen mit Brot stellte sich heraus, dass der Mageninhalt des operierten Hundes eine recht stark ausgeprägte Azidität aufweist. In diesem Zusammenhang stellte Dagajew fest, dass ,,der operierte Magen in einer gewissen Weise die Sekretion der ,transpylorischen Säfte“ regelt."13

Die Brotverdauung verläuft bei fehlendem Magenpförtner wesentlich langsamer als im normalen Zustand. Die Art und Weise der Verdauung der Eiweißstoffe ist sowohl nach der Gastroduodenostomie als auch im Normalfall gleich. Bei dem operierten Hund wurden die Kohlenhydrate dank des Flusses der Säfte aus dem Darm wesentlich rascher verdaut. Bei der Operation nach Billroth-I nähert sich die Magenverdauung bei Brotverzehr der normalen duodenalen Verdauung an.

Die Versuche mit Milch zeigten, dass der Gesamtverlauf der Verdauung bei der Gastroduodenostomie (Billroth-I) um die Hälfte langsamer als normal vonstattengeht. Im Laufe der ersten Stunde tritt Milch gleichmäßig mit allen Komponenten in den Darm über, ohne im Magen zu koagulieren. Die Intensität der Spaltung der gelösten Eiweißstoffe und Kohlenhydrate bleibt konstant, der Umfang der Eiweißverdauung ist wesentlich niedriger als im Normalfall. Die Fette werden wesentlich zügiger gespalten. Der retrograde Übertritt der ,transpylorischen Säfte“ in den Magen ist auf allen Verdauungsstufen zu beobachten und die Reaktion des Magengehalts ist alkalisch.

Die Untersuchung der Verdauung im Darm führte Dagajew bei der Gastroduodenostomie an den Hunden „Bobik“ und „Kaschtan“ durch, denen, wie bereits betont, die Darmfistel $125 \mathrm{~cm}$ oberhalb der Ileozökalklappe angelegt worden war. Der Kontrollhund „Belka“ hatte die gleiche Darmfistel erhalten.

Die Schlussfolgerung, zu der der Verfasser der Dissertation gelangte, läuft auf Folgendes hinaus: Bei den Hunden, die einer Pylorektomie unterzogen worden waren, dauert die Darmverdauung doppelt so lange wie im Normalfall. Die ergiebigste Sekretion fällt in die erste Verdauungsstunde. Die Eiweißverdauung verläuft langsamer als im Normalfall. Bei der Verdauung der Kohlenhydrate wurden keine Abweichungen von der Norm festgestellt. Die Spaltung der Fette bleibt etwas hinter der Norm zurück.

Der Hund „Scholty“ wurde nach dem Bilroth-II-Verfahren mit einer Magenfistel im Fundusbereich operiert.

Die allgemeine Schlussfolgerung, zu der Dagajew gelangte, ist folgende: Der Magen von „Scholty“ befreit sich von der Zuckerlösung um ein Drittel langsamer als der nach dem Billroth-I-Verfahren operierte Magen.

Die Versuche mit Amylodextrin zeigten, dass die Reaktion des Magengehalts alkalisch ist und der Austritt der ,transpylorischen Säfte“ in allen Stadien der Verdauung erfolgte. Die Magenverdauung ähnelte der normalen Verdauung im mittleren Abschnitt des Dünndarms, wo Glykogen im alkalischen Medium konvertiert wird.

\footnotetext{
${ }^{13}$ Anthologie der Geschichte der russischen Chirurgie, Bd. 2, S. 102.
} 
In den Versuchen mit Fett ist die Reaktion des Magengehalts alkalisch. Der nach der Billroth-II-Operation verbleibende Restmagen wird von dem Fett wesentlich langsamer befreit als im Normalfall und wesentlich langsamer als nach der Resektion nach BillrothII. Während der gesamten Verdauung vollzieht sich der Reflux des Inhalts des Zwölffingerdarms, was die alkalische Reaktion des Mageninhalts und die Fettaufspaltung im Magen bedingt. Dieser retrograd verlaufende Prozess ist aber geringer ausgeprägt als nach Anwendung des Billroth-I-Verfahrens. Die Intensität der Fettspaltung ist in den ersten zwei Stunden herabgesetzt, während der weiteren drei Stunden übertrifft sie die Norm und kommt der Intensität nach der Billroth-I-Operation fast gleich.

Die Versuche mit Fleisch zeigten, dass der Magen von „Scholty“ dieses sehr langsam in den Darm transportiert. Beim Verzehr des Hackfleisches gehen binnen drei Stunden der Verdauung nur 17 Prozent in den Darm über. Die Ursache für den langsamen Weitertransport des Fleisches ist die mechanische Insuffizienz des Magens. Darin sieht Dagajew eine gewisse biologische Zweckmäßigkeit: Während des langsamen Transportes des Fleisches in den Darm spaltet es der Magen zugleich weitgehend auf und überlässt dem Darm weniger Arbeit als der normale Magen. Die Reaktion des Mageninhalts blieb bei allen Versuchen säuerlich; das konnte das Ergebnis des fehlenden bzw. unwesentlichen Auftretens des Effekts des retrograden Einströmens der „transpylorischen Säfte“ in den Magen sein. „Es ist eine Tatsache“, fasst Dagajew zusammen, „die insofern interessant ist, als der Reflux vielleicht nicht das Ergebnis neuer anatomischer Verhältnisse ist, sondern dadurch bedingt wird, dass eine gewisse Nahrungsart auch einen gewissen Grad der Sekretion der duodenalen Säfte verursacht."14

Bei den Versuchen mit Brot fällt als erstes die Tatsache auf, dass die Verdauung $14 \mathrm{~h}$ nach dem Beginn der Nahrungsaufnahme zu Ende geht, d. h. sie dauert doppelt so lange wie im Normalfall und zwei Stunden länger als bei der Gastroduodenostomie (Billroth-I). Das beschleunigte Tempo des Übergangs der Nahrung aus dem Magen in den Darm fällt nicht wie sonst üblich auf die dritte bzw. vierte Stunde, sondern auf die achte Stunde. Die Reaktion des Mageninhalts ist sauer. Die Eiweißverdauung fällt etwas geringer aus als im Normalfall sowie nach der Billroth-I-Operation.

Beim Milchverzehr geht der Prozess der Magenverdauung erst nach zwölf Stunden zu Ende. Von den Milchkomponenten verlassen die stickstoffhaltigen Stoffe den Magen am langsamsten. Der Umfang des Abbaus der gelösten Eiweißstoffe ist etwa der gleiche wie auch im Normalfall und nach der Gastroduodenostomie. Der Abbau der Kohlenhydrate verläuft in allen Fällen ungefähr gleich. Der Koeffizient des Fettabbaus ist höher als im Normalfall und der gleiche wie auch bei der Gastroduodenostomie.

Die Versuche mit der Darmfistel am Hund „Werny“ zeigten, dass die Dauer der Verdauung nur die Hälfte der Norm betrug und der Verdauungsdauer bei der Gastroduodenostomie gleich ist. Die Exkretion verläuft während der gesamten Verdauungszeit in gleichmäßigem, verlangsamtem Tempo. Die Eiweiß- und

\footnotetext{
${ }^{14}$ Anthologie der Geschichte der russischen Chirurgie, Bd. 2, S. 123.
} 
Kohlenhydrateverdauung ist etwas geringer als im Normalfall und nach der Billroth-IOperation.

Interessante Ergebnisse erhielt Dagajew während der Versuche am Hund „Maltschik“, bei dem der Magen komplett entfernt, das abdominale Ende der Speiseröhre mit dem Zwölffingerdarm verbunden und die Darmfistel $125 \mathrm{~cm}$ oberhalb der Ileozökalklappe angelegt wurde.

Die Versuche an diesem Hund wurden mit Fleisch durchgeführt - einem Produkt, das hauptsächlich im Magen verdaut wird; mit Fleisch gemischt mit Fett und Speisestärke, die bei Hunden ausschließlich im Magen verdaut werden; sowie mit Milch.

Zeitlich verlief die Fleischverdauung in folgender Reihenfolge: Die besonders ergiebige Sekretion fiel auf das erste Drittel der ersten Stunde, aber gegen Ende der ersten Stunde schwand sie schrittweise. Zu Beginn der zweiten Stunde nahm das Ausmaß der Sekretion erneut zu, dann sank sie wieder. Es ist eine gewisse Ähnlichkeit zwischen der Fleischverdauung bei einem Hund ohne Magen und einem Hund mit einer normalen Fleischverdauung festzustellen.

Bei der Eiweißverdauung sind drei Zeitabschnitte festzustellen. Auf allen Verdauungsstufen werden die Eiweißstoffe ab der ersten Portion im Darm verdaut, wobei diese Verdauung schrittweise verstärkt wird und gegen Ende einer normalen Verdauung nahe kommt.

Bei der Verwendung des Gemisches von Fleisch und Speisestärke verläuft die Verdauung im Darm wie auch beim Verzehr des reinen Fleisches. Der Sekretionsverlauf wird nach der Zusammensetzung des Chymus in drei Perioden unterteilt. Fleisch, das zusammen mit der Speisestärke verzehrt wird, bleibt in der ersten Stunde im Darm und nur kleine Portionen von beiden werden im Darm weitertransportiert. Dank ihrer engen Berührung mit dem Verdauungssaft werden sie im Wesentlichen verdaut. Während der nächsten 100 min strömen dagegen Fleisch und Speisestärke in relativ geringer Menge nach unten durch den Darm und die Nahrung passiert den Darm bis zum oberen Abschnitt des Dünndarms, der durch die Verdauungssäfte wenig betroffen ist. Dank dem Verbleib des Fleisches in den oberen Abschnitten wird es besser verdaut Eiweißstoffe und Kohlenhydrate werden in tiefer liegenden Darmabschnitten abgebaut. Die Resorption der stickstoffhaltigen Stoffe ist wesentlich höher als bei der Ernährung allein mit Fleisch.

Bei der Ernährung des Hundes nach der Gastrektomie mit dem Gemisch aus Fleisch, Speisestärke und Schweinefett endet die Verdauung nach $3 \mathrm{~h} 40 \mathrm{~min}$, wie auch bei der Verwendung des reinen Fleisches. Bei dem Gemisch aus Fleisch und Fett geht die Verdauung nach 4 h 20 min zu Ende. Zunächst wird der flüssige gelbliche Chymus abgesondert und erst 18 min nach dem Beginn der Fütterung werden aus der Fistel einzelne Stückchen Fleisch, die durch die Säfte praktisch nicht verändert sind, abgegeben. Solcher Chymus wird im Laufe von 1 h 20 min abgesondert. Anschließend und bis zum Ende des Versuchs wird der normale Darmchymus abgesondert. Während der Darmexkretion zeigen sich zwei Perioden ganz deutlich: die erste, in der der Chymus 
fast ausschließlich aus den wenig veränderten Fleischteilchen besteht; und die zweite, in der Fleisch nicht mehr deutlich zu erkennen ist und der Chymus dem normalen Darmsaft ähnelt. Während der ersten Hälfte des Versuchs enthält der Chymus in relativ geringer Menge Stickstoff, vorwiegend in Form ungelöster Stoffe. Kohlenhydrate werden gespalten, bis Zucker und Dextrine abgesondert werden. In den letzten zwei Stunden der Exkretion verläuft die Absonderung aller Komponenten mehr oder weniger gleichmäßig, dabei wird Stickstoff vorwiegend in Form gelöster Stoffe abgesondert; die Kohlenhydrate sind alle bis zu Zucker und Dextrinen gespalten. Es wurde genauso viel Stickstoff wie auch im Experiment mit Fleisch resorbiert (12\%); gut wurden Kohlenhydrate (89\%) und teilweise Fette $(13 \%)$ resorbiert.

Bei der Ernährung mit Milch dauerte die Sekretion 2 h 45 min und drei Perioden wurden deutlich voneinander abgegrenzt.

Bei dem normalen Hund „Belka“ wurden unter den gleichen Bedingungen etwa $26 \%$ des Stickstoffs, $46 \%$ der Kohlenhydrate und $85 \%$ der Fette aufgenommen. Ausgehend von diesen experimentellen Daten kommt Dagajew zum Schluss, dass sich bei der Ernährung des Hundes mit Milch nach der Gastrektomie drei deutlich abgrenzbare Zeitabschnitte zeigen: Zunächst wird die nicht koagulierte Milch, dann verkäste Milch und zum Schluss der normale Darmchymus abgesondert. Die besonders ergiebige Sekretion fällt insgesamt auf die erste Verdauungsstunde. Der meiste Stickstoff wird im ersten Drittel der ersten Verdauungsstunde verdaut. Die Kohlenhydrate werden in der zweiten Digestionsperiode zügig verdaut, wenn die verkäste Milch sichtbar wird; die Fettverdauung verläuft gleichmäßig. Die Verdauung der Eiweißstoffe und der Abbau der Fette liegen nahe der Norm.

Die Resorption der wichtigsten Milchkomponenten ist zufriedenstellend und kann als normal bezeichnet werden.

Auf der Grundlage des reichen empirischen Datenmaterials formulierte Dagajew einige allgemeine Schlussfolgerungen, denen eine wichtige Bedeutung für das Verständnis der Rolle des Pylorus bei der gesamten Verdauung und im Hinblick auf die veränderten Magenfunktionen nach Pylorektomie zukommt.

Bei der Lösung der Frage nach der Rolle des Pylorusabschnitts bei der Sekretionsfunktion des Magens war Dagajew von den Schriften solcher Autoren wie K. N. Krschischkowski, G. P. Seleny, W. W. Sawitsch und Gross ausgegangen; aufgrund ihrer Forschungen war bekannt, dass unterschiedliche Nahrungsstoffe und deren Verdauungsprodukte, die in den abgesonderten Teil des Magenbodens eingeführt werden, keine Sekretion des Magensaftes auslösen. Wenn sie aber in die Pars pylorica eingeführt werden, lösen sie die entsprechenden Funktionen über den Magenboden aus.

Die Versuche Dagajews an den Hunden mit dem entfernten Pylorusabschnitt zeigten nun, dass sowohl bei der Magenresektion nach Billroth-I als auch bei der Resektion nach Billroth-II die Magensekretion abnimmt. „Die Magensekretion“, bemerkte er, „lässt nach der Entfernung des Pförtnerteils wesentlich nach: Bei fast allen Versuchen stellten wir die Tatsache fest, dass zu jedem Zeitpunkt der Verdauung im Magen weniger Magensaft 
enthalten ist, als im Normalfall. Das zeigt sich bei den Versuchen mit Brot, vor allem aber mit Milch besonders deutlich. “15

Auf der Grundlage aller durchgeführten Versuche formulierte Dagajew seine Theorie der motorischen Funktionen des Magenpförtners. Laut dieser Auffassung ist dieser ein Schlauch, der sich rhythmisch kontrahiert; mit einem Ende ist er mit dem Magenreservoir verknüpft, dessen Inhalt ,abgesaugt“ wird, und mit peristaltischen Kontraktionen wird dieser Inhalt an das entgegengesetzte Ende transportiert.

„Aber“, betont Dagajew „,da der Pylorusabschnitt oberhalb der Nahrung liegt, die sich auf dem Magenboden befindet, kann man ihn mit einem Transportband vergleichen, das der Weiterbeförderung der Nahrungsmasse dient, die im Fundus liegt. Deshalb spielt der Pylorusteil bei der Entleerung der Nahrung aus dem Magen nicht die Rolle einer regelnden Bremse, sondern eher die eines regelnden Beschleunigers“. ${ }^{16}$

Warum verbleibt die Nahrung nach der Pylorektomie länger als im Normalfall im Magen? Dagajews Versuche zeigten: Wenn der Pförtner mit seinen starken Muskeln vorhanden ist, wird der Widerstand der Darmkontraktion leicht überwunden. Nach einer Pylorektomie wird dieser Widerstand durch die schwache Kontraktion des Fundus ventriculi überwunden.

Der Weitertransport der Nahrung aus einem Magen, der keine Pars pylorica besitzt, ist zum einen durch die peristaltischen Darmkontraktionen bedingt, zum anderen durch den auf den Magenfundus wirkenden Reflex des Zwölffingerdarms, der unter der Einwirkung des sauren Mageninhalts auf das Duodenum entsteht.

Das Fehlen des Pförtners verändert somit nicht nur die Funktionsabläufe im Magen, sondern beeinflusst zugleich die Funktionen des Dünndarms und ändert wesentlich das Zusammenwirken des Darms mit dem Magen.

Bei dem langsamen Transport der Nahrung in den Darm baut der Magen ohne Pylorus die einzelnen Komponenten langsamer ab und leistet gleichzeitig weniger vorbereitende Arbeit für den Darm als der normale Magen. Der Darm korrigiert jene Abweichungen in der Nahrungsverarbeitung, die infolge neuer anatomisch-physiologischer Bedingungen bei der Magenfunktion eingetreten sind. Bei dem Verzehr bestimmter Nahrungsarten wird das Zusammenwirken von Magen und Darm insofern verändert, als die unteren Darmabschnitte eine verstärkte Funktion bei der Kompensation der Defizite in der Verdauung übernehmen, die in den oberen Abschnitten des alimentären Kanals entstanden sind. Auf der Grundlage der durchgeführten Versuche kam Dagajew zu dem Schluss, dass ,der Darm sich an den Verdauungsgrad der Nahrung anpasst, die dorthin aus dem Magen gelangt, die Abweichungen der Magenverdauung korrigiert und den Prozess in den Normbereich bringt. Dementsprechend ändert sich die für die Verdauung im Darm notwendige Zeitspanne; sie dauert doppelt so lange an

\footnotetext{
${ }^{15}$ Anthologie der Geschichte der russischen Chirurgie, Bd. 2, S. 170.

${ }^{16}$ Anthologie der Geschichte der russischen Chirurgie, Bd. 2, S. 158.
} 
wie bei Normverhältnissen; in Details ändert sich das Exkretionsbild, aber der Gesamtchemismus der Verdauung weicht nur geringfügig von der Norm ab. “17

Besonderes Augenmerk richtet Dagajew auf die Anpassung der Magenverdauung an unterschiedliche Nahrungsarten. Es stellte sich heraus, dass bei diesem Prozess strikte Zweckmäßigkeit herrscht und die Magenfunktionen von den anatomischen Verhältnissen, die als Ergebnis der Operation entstanden waren, nicht eindeutig vorbestimmt sind. Bei der Verdauung von Fleisch und Brot bleibt die Reaktion des Mageninhalts immer sauer; bei der Verdauung von Kohlenhydraten und Fetten ist sie immer alkalisch. Dies hängt sowohl vom Grad der Sekretion des Magensaftes als auch vom Ausmaß des Übertritts der „transpylorischen Säfte“ in den Magen ab. Auf der Grundlage seiner experimentellen Daten kommt der Autor der Dissertation zum Schluss, dass ,jedem Nahrungsstoff eine bestimmte Art des Übertritts der ,transpylorischen Säfte` in den Magen entspricht." 18

Der Reflux des duodenalen Inhalts ändert auch den Chemismus der Magenverdauung. Eine ausführliche Analyse der Versuchsdaten zeigte, dass sich mit dem Zurückfließen der „transpylorischen Säfte“ in den Magen Verdauungsprozesse vollziehen, die im Normalfall dem Zwölffingerdarm und dem oberen Abschnitt des Dünndarms bzw. dem mittleren Abschnitt des letzteren eigen sind, nämlich: der Abbau von Kohlenhydraten, die Abspaltung der Fettsäuren von den neutralen Fetten und weitere Abbauprozesse. Die Verdauung der eiweißhaltigen Nahrung verlief bei den Versuchshunden in den Fällen, in denen die Pepsinwirkung durch die Galle und das alkalische Milieu der transpylorischen Säfte unterdrückt wurden, auf Kosten der Wirksamkeit der pankreatischen Fermente.

Das moderne Verständnis der Funktionsregelung des Organismus lässt die Frage nach dem „retrograden Einfluss“ mittels der „Rückreflexe“ auf die Funktionen jener Organe, auf die sie gerichtet sind, bedeutsam erscheinen. „Aus physiologischer Sicht“, hatte Pawlow angemerkt, ,ist folgende Frage äußerst wichtig: Von welcher Stelle des Verdauungskanals aus nimmt die reflektorische Beeinflussung der Magendrüsen ihren Anfang? Ist es nur der Magen selbst oder auch der Darm? Ausgehend von den Daten unserer Versuche muss man sagen, dass dieser Reflex auch von der Schleimhaut des Darms erregt wird."19

Wie wir bereits wissen, wurde der reflektorische Einfluss des Zwölffingerdarms von Dagajew während der Untersuchung der Regelung der Entleerung im normalen und operierten Magen immer berücksichtigt. In seiner Serie wurden die Versuche so durchgeführt, dass die Nahrung nur eine Viertelstunde lang in den Kolben gegossen wurde; während ,der weiteren drei Viertel Stunden kam sie in die unteren Bereiche des Darms,

\footnotetext{
${ }^{17}$ Anthologie der Geschichte der russischen Chirurgie, Bd. 2, S. 177.

${ }^{18}$ Anthologie der Geschichte der russischen Chirurgie, Bd. 2, S. 169.

${ }^{19}$ Pawlow, I. P., Gesammelte Werke, Bd. II, Buch 2, S. 222.
} 
wodurch die Rückwirkung der unteren Bereiche des Verdauungskanals auf den oberen beibehalten wurde. “20

Auf der Basis aller seiner experimentellen Daten gelangte Dagajew zur Schlussfolgerung, dass die Billroth-I-Operation physiologischer als die Billroth-II-Methode ist. Über die letztere schrieb er: „Diese Operation, die die anatomischen Verhältnisse des Verdauungstrakts wesentlich mehr stört, schafft im Organismus offensichtlich nicht besonders günstige Voraussetzungen für dessen Existenz. Es ist wahrscheinlich, dass die verlangsamte Verdauung die Aufnahme und die Verarbeitung jener Tagesmenge an Nahrung nicht zulässt, die hinsichtlich der Deckung des Aufwandes für den Stoffwechsel notwendig ist; mit anderen Worten: Die Verdauung kann mit der inneren Metamorphose der Stoffe nicht Schritt halten. Im Magen bildet sich eine Stase, die bei einer übermäßigen Füllung das Erbrechen verursacht."21

Die Dissertationsschrift von Wladimir Fjodorowitsch Dagajew war nicht nur damals, als er promovierte, innovativ und auch im internationalen Maßstab herausragend und aktuell, sondern sie bleibt es bis heute. Wir werden gewiss wir nichts Falsches sagen, wenn wir uns seinen Worten anschließen, dass die Untersuchung der Verdauungsfunktionen bei den Hunden ,nach der Operation hauptsächlich auf die Untersuchung des Stoffwechsels gerichtet wurde; in der Literatur gibt es keine Versuche, die unseren analog wären."

In diesem Falle geht es um die absolute Priorität der russischen Wissenschaft. Die Arbeit Dagajews wurde sehr umfassend von ausländischen Autoren zitiert.

\footnotetext{
${ }^{20}$ Anthologie der Geschichte der russischen Chirurgie, Bd. 2, S. 177.

${ }^{21}$ Anthologie der Geschichte der russischen Chirurgie, Bd. 2, S. 178-179.
}

Open Access Dieses Kapitel wird unter der Creative Commons Namensnennung 4.0 International Lizenz (http://creativecommons.org/licenses/by/4.0/deed.de) veröffentlicht, welche die Nutzung, Vervielfältigung, Bearbeitung, Verbreitung und Wiedergabe in jeglichem Medium und Format erlaubt, sofern Sie den/die ursprünglichen Autor(en) und die Quelle ordnungsgemäß nennen, einen Link zur Creative Commons Lizenz beifügen und angeben, ob Änderungen vorgenommen wurden.

Die in diesem Kapitel enthaltenen Bilder und sonstiges Drittmaterial unterliegen ebenfalls der genannten Creative Commons Lizenz, sofern sich aus der Abbildungslegende nichts anderes ergibt. Sofern das betreffende Material nicht unter der genannten Creative Commons Lizenz steht und die betreffende Handlung nicht nach gesetzlichen Vorschriften erlaubt ist, ist für die oben aufgeführten Weiterverwendungen des Materials die Einwilligung des jeweiligen Rechteinhabers einzuholen. 